University of Louisville

ThinkIR: The University of Louisville's Institutional Repository

Faculty Scholarship

$1-2014$

\title{
Testing three different sequential mediational interpretations of Beck's cognitive model for the development of depression.
}

\author{
Patrick Pössel \\ University of Louisville \\ Stephanie Winkeljohn Black \\ University of Louisville
}

Follow this and additional works at: https://ir.library.louisville.edu/faculty

Part of the Cognitive Psychology Commons, and the Counseling Psychology Commons

\section{Original Publication Information}

This is the peer reviewed version of the following article:

Pössel, Patrick and Stephanie Winkeljohn Black. "Testing Three Different Sequential Mediational Interpretations of Beck's Cognitive Model of the Development of Depression." 2014. Journal of Clinical Psychology, 70(1): 72-94.

which has been published in final form at https://doi.org/10.1002/jclp.22001

This article may be used for non-commercial purposes in accordance with Wiley Terms and Conditions for Self-Archiving.

This Article is brought to you for free and open access by ThinkIR: The University of Louisville's Institutional Repository. It has been accepted for inclusion in Faculty Scholarship by an authorized administrator of ThinkIR: The University of Louisville's Institutional Repository. For more information, please contact thinkir@louisville.edu. 
Testing Three Different Sequential Mediational Interpretations of Beck’s Cognitive Model of the Development of Depression

\section{Patrick Pössel}

Department of Clinical and Developmental Psychology, Eberhard-Karls-University, Tübingen,

\section{Germany}

Stephanie Winkeljohn Black

Department of Educational and Counseling Psychology, University of Louisville, Louisville,

$$
\text { KY, USA }
$$

Acknowledgments

Patrick Pössel is now Associate Professor in the Department of Educational and Counseling Psychology at the University of Louisville.

Corresponding author:

Patrick Pössel, Dr. rer. soc.

Dept. of Educational and Counseling Psychology

College of Education and Human Development

University of Louisville

2301 S. Third Street

Louisville, KY 40292

USA

Office: (502) 852-0623

Fax: (502) 852-0629

E-mail: patrick.possel@louisville.edu 


\begin{abstract}
Objectives: This study tested and compared three sequential interpretations of Beck's cognitive model of the development of depression (1996). The causal mediational interpretation identifies dysfunctional attitudes as most distal to depressive symptoms, followed by cognitive distortions, cognitive triad, and negative automatic thoughts, with each construct successively more proximal to depressive symptoms. By contrast, the symptom model reverses the causal chain with negative automatic thoughts as the most proximal consequence and dysfunctional attitudes as the most distal consequence of depression. The bidirectional model merges both interpretations into one model. Previous studies on sequential interpretations of Beck's model have not included cognitive distortions and the cognitive triad and did not test the bidirectional model finding contradictory empirical evidence for the sequential order. Method: In the 3-wave longitudinal study, 308 German university students without clinically significant depressive symptoms (245 female, average age: 23.69 years) completed self-report questionnaires measuring their dysfunctional attitudes, cognitive distortions, cognitive triad, negative automatic thoughts, and depressive symptoms. Results: The bidirectional model with partial mediation fit the data best and cognitive distortions mediated the relationship between dysfunctional attitudes and negative automatic thoughts and vice versa. Conclusions: The findings have important consequences for the prevention of depression. Prevention programs may want to focus on cognitive distortions, the only construct in Beck's model that influences every other construct in the model.
\end{abstract}

Keywords: Beck's cognitive model of depression; causal mediation; sequential model; symptom model; mood-state hypothesis. 
Beck's cognitive model of depression (1976) has emerged as one of the major cognitive theories to explain the development and maintenance of depression and has formed the basis of one of the most effective approaches to treating depression (Butler, Chapman, Forman, \& Beck, 2006; Pössel \& Hautzinger, 2006). Within Beck’s model (1976), schemas, negative dysfunctional attitudes, cognitive distortions, the cognitive triad, and negative automatic thoughts are central constructs. Schemas form the cognitive structure that organizes and help interpret information while attitudes are the constent of these schemas. Attitudes are relatively enduring, organizing structures that guide situational information processing. Dysfunctional attitudes are often negative in content and consist of immature, absolute, and rigid attitudes about the self and its relation to the world. When activated by stress, negative dysfunctional attitudes (hereafter referred to as "dysfunctional attitudes") lead to cognitive distortions, which cause perception and thinking to be unrealistic, extreme, and distorted in a negative way. As a result, thinking is dominated by a negative view of the self, the world, and the future- the cognitive triad. According to Beck, the cognitive triad is expressed through negative automatic thoughts temporary, non-emotional mental events that may be subjectively plausible in certain situations which influence emotional, somatic, and motivational symptoms of depression.

Beck's cognitive model can be interpreted as a causal mediational model (Alloy, Clements, \& Kolden, 1985), with the cognitive constructs of the model organized based on their sequential relationships to depressive symptoms. In this sequence, dysfunctional attitudes are seen as the most distal construct, followed by cognitive distortions, the cognitive triad, and then negative automatic thoughts as the most proximal construct with regard to depressive symptoms. In other words, as the most distal construct in the causal mediational model, dysfunctional attitudes trigger a chain of cognitive variables that finally lead to emotional, somatic, and 
motivational symptoms of depression. Additionally, the model assumes that each cognitive construct fully mediates the relationship between its preceding and subsequent constructs. For example, dysfunctional attitudes do not contribute directly to the cognitive triad, negative automatic thoughts, and depressive symptoms, but are instead mediated through cognitive distortions (see Figure 1, top graph).

The symptom model is another interpretation of Beck's cognitive model (see Figure 1, middle graph; Brewin, 1985). The symptom model assumes that cognitive constructs reflect changes in depressive symptoms and have no causal impact on the development and maintenance of depressive symptoms. Furthermore, in this model, the causal chain is reversed with negative automatic thoughts as a proximal consequence of depression and dysfunctional attitudes as the distal consequence (Kwon \& Oei, 1992; Parry \& Brewin, 1988). In other words, contrary to the causal mediational model, the symptom model proposes that emotional, somatic, and motivational symptoms of depression trigger a chain of cognitive variables.

The causal mediational and symptom models can be combined into a bidirectional model (see Figure 1, bottom graph). Beck (1967, 1996; Beck \& Weishaar, 2005) himself assumed bidirectional effects between cognitive constructs and depressive symptoms. Beck (1967) proposed that the activation of cognitive constructs causes the development of depressive symptoms (top-down processes), including negative emotions, which in turn further innervate and consequently reinforce already existing dysfunctional attitudes (bottom-up influences). In an update to his traditional theory, Beck (1996; Beck \& Weishaar, 2005) introduced the concept of modes. Modes represent a network of cognitive, affective, motivational, and behavioral components that are designed to deal with specific demands or problems. Although cognitive schemata reside at the center of a given mode, all other components of a mode (affective, 
motivational, and behavioral) influence the cognitive schemata as they in turn influence the other components.

There is mixed empirical evidence for both the causal meditational and symptom model interpretations of Beck's cognitive model. In a cross-sectional study with undergraduate students, Kwon and Oei (1992, Study 1) found support for the causal mediational interpretation of Beck's cognitive model concerning dysfunctional attitudes, negative automatic thoughts, and depressive symptoms. However, their data also supported the symptom model. In a 2-wave longitudinal study with undergraduates, Kwon and Oei (1992, Study 2) replicated support for both models. In a 2-wave longitudinal study focusing on cognitive distortions and depression in adolescents from Hong Kong and the United States, Stewart et al. (2004) found that both the causal mediational model and the symptom model were supported by data from the two samples.

Contrary to the above results, other cross-sectional studies measuring dysfunctional attitudes, negative automatic thoughts, and depressive symptoms supported the causal mediational model but not the symptom model (Oei, Goh, \& Kwon, 1996; Oei \& Kwon, 2007) or the symptom model but not the causal mediational model (Oei, Hibberd, \& O'Brien, 2005). Further, a 2-wave longitudinal study from Joiner et al. (1999) found support for the causal mediational model but did not test the symptom model. Finally, another 2-wave longitudinal study with outpatients treated with cognitive-behavioral therapy (CBT) found that dysfunctional attitudes at baseline did not predict depression 12 weeks later or vice versa (Burns \& Spangler, 2001). Therefore, Burns and Spangler's (2001) study found no support for the causal mediational, the symptoms model, or the bidirectional model. However, as the participants were treated with CBT between each wave, it is likely that the associations between dysfunctional 
attitudes and depression were underestimated. Overall, previous studies do not provide a clear picture as to which model is most applicable to Beck's theory.

Three of the eight studies which tested both models found support for both models, three studies found support for the mediational model only, one study supported only the symptom model, and one study supported neither of the models. One reason that may explain the inconsistent results is that although the causal mediational and the symptom model were both tested in seven of the eight studies described here, the bidirectional model was tested in only one study. While this study (Burns \& Spangler, 2001) did not find support for the bidirectional model, it is likely the associations between the variables in Beck's cognitive model (1967, 1996; Beck \& Weishaar, 2005) were underestimated due to the systematic intervention which occurred between the waves.

Additionally, seven of the eight studies did not differentiate between individuals with and without clinically significant depressive symptoms, nor did they describe the severity of depressive symptoms in their samples. However, the symptom model is based on the assumption that existing depressive symptoms trigger the activation of the cognitive constructs (Beck, 1967; Kwon \& Oei, 1992; Parry \& Brewin, 1988). Thus, while the causal mediational and bidirectional models may better explain the development of depressive symptoms, the symptom model may better explain the maintenance of depressive symptoms. As such, differences in the depressive symptomatology of the samples may explain these studies' inconsistent results.

Finally, four of the eight studies were cross-sectional and the remaining four studies applied a 2-wave longitudinal design. It is widely known that causal conclusions cannot be drawn from cross-sectional studies. Cross-sectional studies are even less sufficient to provide evidence about mediations (Cole \& Maxwell, 2003). Therefore, the four cross-sectional studies 
are not sufficient to test the sequential interpretations of Beck's cognitive model $(1967,1996$; Beck \& Weishaar, 2005). When testing mediation models, 2-wave longitudinal studies have serious limitations as well. Mediation models propose three specific causal relationships between variables (independent variable - dependent variable, independent variable - mediator variable, mediator variable - dependent variable). However, if data are collected at only two time points, one of these relationships has to be cross-sectional. Therefore, no causal conclusions about this relationship can be drawn, which is the reason why Cole and Maxwell (2003) have called 2-wave studies intended to test mediation models "half-longitudinal." The authors further concluded that 3-wave longitudinal studies are necessary in order to make inferences about all three relationships in a mediation model.

Sequential interpretations of Beck's cognitive model assume that each cognitive construct fully mediates the relationship between its preceding and subsequent constructs. However, the literature does not support this assumption. The studies that tested mediation effects found that negative automatic thoughts were only partial mediators (Kwon \& Oei, 1992; Oei et al., 1996). In other words, while negative automatic thoughts mediated the relationship between dysfunctional attitudes and depressive symptoms, there were still significant direct relationships between dysfunctional attitudes and depressive symptoms. Another limitation in the literature about the sequential interpretations of Beck's cognitive model is that none of the studies that tested the sequential interpretation included cognitive distortions or the cognitive triad. Not only did this limit the studies' overall ranges of interpretability, but especially it especially limited their ability to determine whether the cognitive constructs completely mediate the association between their preceding and subsequent constructs. 
Thus, the empirical literature on Beck's cognitive model of depression (1967, 1996; Beck \& Weishaar, 2005) is limited in several ways. First, the bidirectional model has been tested in only one study (Burns \& Spangler, 2001) and this study likely underestimated the associations between variables in Beck's cognitive model, causing a lack of support for all three hypothezised models. Second, the studies did not differentiate between participants with and without clinically significant depressive symptoms. Third, it is unclear if the different cognitive constructs acted as full (no significant relationship between independent and dependent variable are left after inclusion of the mediator variable) or partial mediators (independent and dependent variable are still directly related after inclusion of the mediator variable) within the models. Fourth, none of the existing studies included all of the cognitive constructs of Beck's model (dysfunctional attitudes, cognitive distortions, cognitive triad, and negative automatic thoughts).

The current study sought to overcome these limitations by testing the three interpretations of Beck's cognitive model of depression: the causal mediational model, the symptom model, and the bidirectional model. The study examined dysfunctional attitudes, cognitive distortions, the cognitive triad, negative automatic thoughts, and depressive symptoms in the same sample from the general population. All three models were tested as both full and partial mediational models. In addition, the models were tested only in participants without preexisting clinically significant depressive symptoms. It was expected that the causal mediational or the bidirectional interpretation of Beck's cognitive model (Beck, 1967, 1996; Beck \& Weishaar, 2005) would be confirmed for these individuals. Furthermore, based on the literature (Kwon \& Oei, 1992; Oei et al., 1996) it was expected that a model allowing for partial mediation would fit the data better than a full mediation model.

\section{Method}




\section{Participants}

The sample consisted of 398 (319 female, 79 male) German psychology students at a university in southwest Germany. As expected in a college student sample (Wittchen, Nelson, \& Lachner, 1998), 90 (22.6\%) participants reported clinically significant depressive symptoms in a self-report measure. These students were excluded from the analyses. The remaining 308 students without clinically significant depressive symptoms ranged in age from 18 to 52 years $(\mathrm{M}=23.69, \mathrm{SD}=6.12$ years $)$ and included 245 female $(79.5 \%)$ and 63 male $(20.5 \%)$ students. Out of the total of 308 students, 39 (29 female, 10 male) dropped out between the first (T1) and second time points (T2; 269 remaining); an additional 23 students (18 female, 5 male) dropped out between the second and third time point (T3; 246 remaining). There were no differences between the dropouts and the remaining students in sex, $\chi^{2}(1)=1.13, p=.287$, or depressive symptoms, $t(308)=-0.69, p=.494$. However, dropouts were significantly older, $t(308)=-2.02$, $p=.044$, than remaining students.

\section{Measures}

\section{Center for Epidemiological Studies - Depression Scale (CES - D). The CES-D}

(Radloff, 1977; German version: Hautzinger, Bailer, Hofmeister, \& Keller, 2012) consists of 20 items (e.g., "I was bothered by things that usually don't bother me.") and is a quickly administered, economic screening instrument that measures depressive symptoms based on selfreport. Frequency of symptoms is rated on a 4-point Likert scale, with higher numbers indicating a higher frequency of occurrence. The scale has a range from 0 to 60 . A score of $\geq 23$ represents clinically significant depressive symptoms (Hautzinger et al., 2012). Studies with the original and the German version of the CES-D demonstrate high sentitivity (82-85\%) and specificity (84-94\%) (Chiu et al, 2010; Haringsma, Engels, Beekman, \& Spinhoven, 2004; 
Hautzinger et al., 2012). The CES-D scores have internal consistencies of $\alpha=.89$ to .92 in samples from the German general population. The CES-D scores showed an internal consistency of $\alpha=.90$ in the present study.

Dysfunctional Attitudes Scale (DAS). The DAS Form A (Weissman \& Beck, 1978; German version: Hautzinger, Joormann, \& Keller, 2005) consists of 40 items (e.g., "People will probably think less of me if I make a mistake”) on a 7-point Likert scale to measure depressogenic schemata, a cognitive construct described by Beck (1976). Higher scores represent a greater endorsement of dysfunctional beliefs. The internal consistencies of the DAS in German samples reported by Hautzinger et al. (2005) ranged from $\alpha=.88$ to .94 . In the present study, the internal consistency was $\alpha=.88$.

Cognitive Error Questionnaire (CEQ). The CEQ (Lefebvre, 1981; German version: Pössel, 2009a) consists of 24 items (e.g., "You just finished spending three hours cleaning the basement. Your spouse however, doesn't say anything about it. You think to yourself, 'S/He must think I did a lousy job."') on a 5-point Likert scale to measure cognitive distortions, as described by Beck (1976).The CEQ includes the subscales "catastrophizing," "overgeneralization," "personalization," and "selective abstraction." All item values are summed, where higher scores represent greater endorsement of cognitive distortions. The internal consistency for the CEQ total score in the German validation sample had a Cronbach's $\alpha$ of .87. Further, the internal consistency in this sample was $\alpha=.85$.

Cognitive Triad Instrument (CTI). The CTI (Beckham, Leber, Watkins, Boyer, \& Cook, 1986; German version: Pössel, 2009b) measures the cognitive triad, as described by Beck (1976). The CTI includes three subscales which measure the view of the self (10 items), the world (10 items), and the future (10 items) on a 7-point Likert scale (e.g., "There is nothing left in my life 
to look forward to.”). There are six additional items that are unscored filler items. Items are phrased in both positive and negative directions. Therefore, before calculating the scores for the CTI total scale by summing, all items have to be pooled so that higher scores represent positive views and low scores represent negative views. In the German validation sample, the internal consistencies of the CTI total score was $\alpha=.88$ (Pössel, 2009b). In the present study, the internal consistency of the CTI total score was $\alpha=.89$.

Automatic Thoughts Questionnaire-Revised (ATQ-R). The ATQ-R (Kendall, Howard, \& Hays, 1989; German version: Pössel, Seemann, \& Hautzinger, 2005) measures automatic thoughts, as described by Beck (1976). The German ATQ-R includes the subscales "negative self-statements" (12 items), "well-being” (5 items), and "self-confidence” (4 items) on a 5-point Likert scale (e.g., "No one understands me."). A higher summary score in the subscale "negative self-statements" indicates more negative automatic thoughts, whereas higher scores in the subscales "well-being" and "self-confidence" indicate more positive automatic thoughts. The internal consistency for the negative self-statements scale was $\alpha=.86$ in the German sample from the common population, whereas the internal consistencies for the well-being and selfconfidence scales were only $\alpha=.76$ and .67 , respectively. Furthermore, the ATQ-R scales correlated with the German CES-D to $r=.71,-.41$, and -.11. Thus, the negative self-statements scale is not only more reliable than the two positive scales but also correlates significantly higher with depression (Pössel et al., 2005). Therefore, only the negative self-statements scale was administered in the present study. In this study, the negative self-statement subscale showed a good internal consistency $(\alpha=.89)$.

\section{Procedures}


Participants were recruited with a notice posted on the university campus; the notice stated that standardized questionnaires would be used to study the association between cognitions and emotions. Participants in groups of 8 to 15 completed questionnaire batteries at the beginning (T1), middle (T2), and end (T3) of the semester (each 4 weeks apart). The order of the questionnaires was counterbalanced across the sample following the Latin square design. Informed consent was obtained for all participants, who received academic credits for their participation. The study was approved by the ethical committee of the German Psychological Association.

\section{Data Analysis}

The tests of the hypothesized mediation models followed Cole and Maxwell's (2003) approach for 3-wave studies using structure equation models and Martens and Haase's (2006) suggestion on how to compare different models. The analyses were conducted with the maximum likelihood method using AMOS 19.0 to calculate structural equation models (Arbuckle, 1999). Models' goodness of fit was tested with $\chi^{2}$. Because the $\chi^{2}$ value tends to be substantial and statistically significant when the model does not hold and the sample size is large (Joreskog \& Sorbom, 1993), the $\chi^{2}$ was complemented with $\chi^{2} / d f$, the root mean square error of approximation (RMSEA; Steiger \& Lind, 1980), the Tucker-Lewis Index (TLI; Tucker \& Lewis, 1973), and the comparative fit index (CFI; Bentler, 1990). Nonsignificant $\chi^{2}$ values and $\chi^{2} / d f$ values under 2 are preferred. Regarding the CFI and TLI, values > .95 indicate good model fit and values > .90 are acceptable. RMSEA values <.05 demonstrate good model fit and values $<.08$ are acceptable (Hu \& Bentler, 1999).

To identify the best model, the data were first fit to (a) an autoregressive model (only the regressions between each variable at one time point and the same variable at the next time point 
are allowed to vary freely, all other regressions are set to 0; Figure 2, top left graph),(b) the fully mediated causal mediational (in addition to the the regressions allowed to vary freely in the autoregressive model, only the regressions between each variable at one time point and the variable right after this variable in the theoretical sequence at the following time point are allowed to vary freely, all other regressions are set to 0; Figure 2, top right graph), (c) the fully mediated symptom models (in addition to the regressions allowed to vary freely in the autoregressive model, only the regressions between each variable at one time point and the variable right before this variable in the theoretical sequence at the following time point are allowed to vary freely, all other regressions are set to 0; Figure 2, bottom left graph), (d) the fully mediated bidirectional model (in addition to the regressions allowed to vary freely in the autoregressive model, only the regressions between each variable at one time point and the variable right before and after this variable in the theoretical sequence at the following time point are allowed to vary freely, all other regressions are set to 0; Figure 2, bottom right graph), (e) the partially mediated causal mediational (in addition to the regressions allowed to vary freely in the autoregressive model, only the regressions between each variable at one time point and all variables after this variable in the theoretical sequence at the following time point are allowed to vary freely, all other regressions are set to 0 ; Figure 2, top right graph), (f) the partially mediated symptom models (in addition to the regressions allowed to vary freely in the autoregressive model, only the regressions between each variable at one time point and all variables before this variable in the theoretical sequence at the next time point are allowed to vary freely, all other regressions are set to 0 ; Figure 2, bottom left graph), and (g) the partially mediated bidirectional (fully cross-lagged) model (all regressions between any variable at one time point and all variables at the following time point are allowed to vary freely). Then the autoregressive model 
was compared with the causal mediational model and the symptom model. Next, the model fitting the data best was compared with the bidirectional model (Martens \& Haase, 2006).

This procedure allowed the usage of $\chi^{2}$ difference tests because all but the causal mediational and symptom models were nested in the previously tested models. In $\chi^{2}$ difference tests, the $\chi^{2}$ values as well as the $d f$ s of the models are subtracted from each other. When $\Delta \chi^{2}$ is significant for $\Delta d f$, the models are seen as significantly different from each other. In addition, the CFI of the calculated models were used to compare the models. $\triangle \mathrm{CFI}$ is calculated by subtracting the CFI value of one model from the CFI value of another model. When $\triangle \mathrm{CFI}$ of two models is $>.002$ the model with higher CFI fits the data significantly better. However, when $\Delta \mathrm{CFI}$ is $\leq .002$ both models fit equally well from a statistical point of view and the more parsimonious model should be accepted (Meade, Johnson, \& Braddy, 2008). This procedure to compare models was repeated for models with full and partial mediation.

In order to test for multiple mediators, the approach of Preacher and Hayes (2008) was followed by calculating $95 \%$ bootstrapping confidence intervals (CI) using the bias-corrected percentile method. Following this approach, multiple mediation effects exist when the indirect effect (effect from independent variable through all possible mediators to dependent variable) is significant. However, as this procedure tests only for multiple mediation effects, $95 \%$ confidence intervals for the hypothesized individual mediation effects were calculated using PRODCLIN (MacKinnon, Fritz, Williams, \& Lockwood, 2007). The upper and lower confidence limits have different critical values because PRODCLIN uses the product method, which follows an asymmetrical distribution (MacKinnon et al., 2007). A statistically significant mediation effect exists when the confidence interval does not contain zero.

\section{Results}


Descriptive data and correlations for all instruments are presented in Table 1. All measures were moderately to highly correlated with each other. Nevertheless, the correlations did not signal potential problems with multicollinearity (Kline, 2005). The manifest variables had an approximately normal distribution (Chou \& Bentler, 1995; Kline, 2005).

\section{Determination of the Best Fitting Model}

Six theory-driven interpretations of Beck's cognitive model of depression and an autoregressive model were fit to the data $(N=235$; see Table 2$)$. First, the models with full mediation were tested and compared using the $\chi^{2}$ difference test and the $\Delta$ CFI. Comparisons of the autoregressive model with the causal mediational, $\Delta \chi^{2}(8, N=299)=18.52, p<.05, \Delta \mathrm{CFI}=$ .004 , and the symptom model, $\Delta \chi^{2}(8, N=299)=22.16, p<.01, \Delta \mathrm{CFI}=.005$, revealed that both theory-driven models provided a significantly better fit to the data than the autoregressive model. Thus, the causal mediational, $\Delta \chi^{2}(8, N=299)=23.91, p<.01, \Delta \mathrm{CFI}=.006$, and the symptom model, $\Delta \chi^{2}(8, N=299)=20.27, p<.01, \Delta \mathrm{CFI}=.005$, were compared to the bidirectional model. Results of the $\chi^{2}$ difference tests and the $\Delta$ CFI's indicated that the bidirectional model with full mediation fit the data better than the two other theory-driven interpretations of Beck's cognitive model.

Next, the models with partial mediation were tested and compared. Comparing the autoregressive model with the causal mediational, $\Delta \chi^{2}(20, N=299)=71.84, p<.001, \Delta \mathrm{CFI}=$ .019 , and the symptom model, $\Delta \chi^{2}(20, N=299)=55.90, p<.001, \Delta \mathrm{CFI}=013$, revealed that both theory-driven models provided a significantly better fit to the data than did the autoregressive model. Thus, the causal mediational, $\Delta \chi^{2}(20, N=299)=54.57, p<.001, \Delta \mathrm{CFI}=$ .012 , and the symptom model, $\Delta \chi^{2}(20, N=299)=70.51, p<.001, \Delta \mathrm{CFI}=.018$, were compared with the bidirectional model. Results of the $\chi^{2}$ difference tests indicated that the bidirectional 
model with partial mediation fit the data better than the two other theory-driven interpretations of Beck's cognitive model with partial mediation.

Finally, the two bidirectional models with full and partial mediation, respectively, were compared using the $\chi^{2}$ difference test, $\Delta \chi^{2}(24, N=299)=83.98, p<.001, \Delta \mathrm{CFI}=.021$, revealing that the bidirectional model with partial mediation fit the data best. Nevertheless, it should be mentioned that the bidirectional model with partial mediaton was superior to the other tested models in only two (CFI) of the five calculated indices, and only the CFI of this model was acceptable. Finally, the $\chi^{2} / d f$ of 5.74 provided further support for the idea that the bidirectional model can be improved to better fit the data.

The standardized regression weights of the bidirectional model are presented in Figure 3 and the correlations between scales at the same time point are presented in Table 3 . Some specifics should be noted. First, the correlations (Table 3) demonstrate that nearly every scale correlated significantly with all other scales at the same time point. The correlations between ATQ and CES-D at each time point are the highest correlations, ranging from $r=.567$ to .695 . Second, each scale at one time point significantly predicted the same scale at a later time point (Figure 3). Third, of 20 additional regressions (i.e., not autoregressive) predicted by the causal meditational model, only 7 were significant or marginally significant. Further, of 20 additional regressions predicted by the symptom model, only 2 were significant or marginally significant (ATQ at T1 predicting CEQ at T2, CEQ at T2 predicting DAS at T3). Although proposed by the bidirectional model, the CES-D did not predict a score in any instrument measuring a cognitive construct. Thus, bidirectional relations existed only between different instruments measuring cognitive constructs. Fourth, at any time point CES-D is affected by all cognitive scales but the ATQ. Fifth, the patterns of significant nonautoregressive regressions were different between T1 
- T2 and T2 - T3. Although DAS, CTI, and ATQ scores at T1 had nonautoregressive effects on scales at T2, at T2, none of these instruments had any nonautoregressive effect on scales at T3. The CEQ scores demonstrated a pattern of regressions opposite to the pattern of the DAS, CTI, and ATQ. CEQ at T1 predicted only CEQ at T2, but CEQ at T2 predicted scores of all scales but the CTI at T3. Sixth, the pattern of regressions that are significant or marginally significant revealed that the CEQ was central to all possible mediation effects: (a) The effect of DAS at T1 on ATQ and CES-D at T3 can be mediated by CEQ at T2; and (b) the effect of ATQ at T1 on DAS at T3 can be mediated by CEQ at T2.

\section{Tests for Mediation.}

In order to test for multiple mediation effects, $95 \%$ bootstrapping confidence intervals were calculated and presented in Table 4 . These analyses revealed that multiple mediation effects between each construct at T1 and the same construct at T3 existed. Further and more relevant for the hypotheses, multiple mediation effects existed between DAS at T1 and ATQ as well as CES-D at T3, between CEQ at T1 and DAS as well as ATQ at T3, between CTI at T1 and DAS as well as CES-D at T3 and between ATQ at T1 and CEQ as well as DAS at T3.

Based on the findings regarding multiple mediation effects, asymmetrical $95 \%$

confidence intervals for the hypothesized individual mediation effects in the bidirectional model allowing for partial mediation were calculated and presented in Table 5. Only the confidence intervals for the following three mediated relationships did not contain zero and were therefore significant: DAS at T1 - CEQ at T2 - ATQ at T3, DAS at T1 - CEQ at T2 - CES-D at T3, and ATQ at T1 - CEQ at T2 - DAS at T3. Thus, all tested regressions between DAS, ATQ, and CES-D were mediated by CEQ at T2. 


\section{Discussion}

The primary aim of this study was to test and compare different interpretations of Beck's cognitive model of the development of depression (Beck, 1967, 1996; Beck \& Weishaar, 2005). Based on theoretical considerations (Beck, 1967, 1996; Beck \& Weishaar, 2005) and empirical literature (Kwon \& Oei, 1992; Oei \& Kwon, 2007; Stewart et al., 2004), it was proposed that either the causal mediational or bidirectional model interpretations of Beck's cognitive model would be confirmed for individuals without clinically significant depressive symptoms. It was further expected that models allowing for partial mediation would fit the data better than full mediation models.

The study had three main results. First, consistent with the hypotheses, the model with partial mediation fit the data best in individuals without clinically significant depressive symptoms at Time 1. Specifically, the bidirectional model with partial mediation fit the data best. However, it should be mentioned that the goodness of fit indices demonstrate that the bidirectional model did not fit the data well. This might be caused by the second main finding. While most of the cognitive variables influenced each other, and although depressive symptoms were influenced by all cognitive variables with the exception of negative automatic thoughts, depressive symptoms predicted only one cognitive variable (cognitive distortions at Time 3). Thus, in individuals without clinically depressive symptoms, cognitive constructs influenced each other and each other's impact on depressive symptoms. In other words, the findings did not support the concept of distal and proximal variables in relation to depressive symptoms in individuals without clinically significant depressive symptoms. Instead, the bidirectional relations highlighted the flexibility of cognitive constructs in such individuals. The third main finding was that all tested mediation effects were significant, with cognitive distortions 
mediating the effect of dysfunctional attitudes on negative automatic thoughts and depressive symptoms as well as the effect of negative automatic thoughts on dysfunctional attitudes. It is possible that the impression of bidirectional relationships in individuals without significant depressive symptoms at the beginning of the study might have been caused by a blending of topdown processes and bottom-up influences (Beck, 1967). A differentiation between top-down processes and bottom-up influences can be drawn with the first being seen as dysfunctional attitudes causing negative automatic thoughts while in the latter negative automatic thoughts only activate existing dysfunctional attitudes. Two experimental studies, one with participants with a SCID-I diagnosis of an episode of Major Depression and one with participants without any SCID-I diagnosis, designed to trigger only top-down processes found effects of attitudes on attitudes, thoughts, and emotions while thoughts and emotions showed no effect on attitudes (Pössel \& Knopf, 2008). It could be assumed that the impression of bidirectional effects in longitudinal studies is caused by the fact that top-down processes and bottom-up influences are not separable. Further experimental studies are necessary to test this.

While it was unexpected that cognitive distortions were the sole mediators, this is consistent with Ilardi and Craighead (1999), who conceptualized changing cognitive distortions as a primary mechanism of change. Ilardi and Craighead based their assertion on the assumption that changing cognitive distortions would ultimately modify dysfunctional attitudes as well. Thus, if replicated, the reported finding supports Ilardi and Craighead's (2009) argument and makes cognitive distortions a core element of Beck's cognitive model of the development of depression (Beck, 1967, 1996; Beck \& Weishaar, 2005).

An unexpected result is that within the model, different associations were significant from Time 1 - Time 2 than from Time 2 - Time 3. This result might be evidence for nonlinear 
relations (Cole \& Maxwell, 2003) that can be caused by a violation of the stationarity assumption. Kenny (1979) noted that stationarity "refers to an unchanging causal structure" (p. 232). Applied to the current study, the stationarity assumption implies that the degree to which dysfunctional attitudes produce changes in cognitive distortions remains the same over time. In other words, it is possible that the differences in significant regressions from Time 1 to Time 2 compared to Time 2 to Time 3 might refer to an acceleration or deceleration of causal relations between the studied constructs. Another explanation for the differences in significant regressions between the waves is that the optimal time between two time points (time lag) may have varied from one part of the model (e.g., dysfunctional attitudes to cognitive triad) to another part of the same model (e.g., cognitive triad to depressive symptoms). For example, Hollon, DeRubeis, and Evans (1996) suggested that while dysfunctional attitudes are relatively stable over time, negative automatic thoughts fluctuate on a moment-to-moment basis. This is supported by Pössel and Knopf (2008), who argued that the activation of dysfunctional attitudes triggers negative automatic thoughts within seconds, which cause immediately depressed mood. Thus, the selected time lag of four weeks between waves may not have been optimal to represent the full causal effect of one variable on another (Cole \& Maxwell, 2003). Nevertheless, it is noteworthy that the bidirectional model's fit was good. Thus, if a violation of the stationarity assumption caused the previously described problems, this violation seemed to have had limited impact on the model fit.

Considering the optimal lengths of the time lag, previous longitudinal studies used time lags between two weeks (Joiner et al., 1999) and six months (Oei \& Kwon, 2007; Stewart et al., 2004) and measured dysfunctional attitudes, negative automatic thoughts, and depressive symptoms. Thus, with a time lag of four weeks, the current study was well within the range 
established by previous studies. Nevertheless, it should be noted that all of the cited longitudinal studies were limited to two waves; they were thus not able to detect if causal relations within Beck's cognitive model (Beck, 1976, 1996; Beck \& Weishaar, 2005) accelerate or decelerate. Future research is needed with least four waves to estimate the optimal time lag between measurements and to test the stationarity assumption.

Besides being the first comprehensive study to test multiple causal interpretations of Beck's (1976, 1996; Beck \& Weishaar, 2005) cognitive model of the development of depression using all of the cognitive variables, the present study also stood out with its relatively large sample size and longitudinal design. However, it is not without limitations. It is unclear whether the stationarity assumption is true for the sequential interpretation of Beck's cognitive model (Beck, 1976, 1996; Beck \& Weishaar, 2005); the nonsignificance of specific regressions in this study should be interpreted cautiously.

While this is the first study testing Beck's cognitive model (Beck, 1976, 1996; Beck \& Weishaar, 2005) using a 3-wave longitudinal design which, allows inferences about all three relationships in a mediation model, one might argue that a 5-wave longitudinal design is necessary to test Beck's model as it includes five elements (dysfunctional attitudes, cognitive distortions, cognitive triad, negative automatic thoughts, and depressive symptoms). Thus, the findings of the current study should be considered with this limitation in mind.

As mentioned above, the goodness of fit indices TLI and RMSEA demonstrated that the bidirectional model does not fit the data well. However, Hu and Bentler (1998) point out that goodness of fit indices are better at distinguishing between models that have different degrees of misspecification than providing absolute guidelines about the acceptability of a particular model. Thus, Marsh, Hau, and Wen's (2004) recommended using the indices to compare the fit of the 
alternative models rather than as absolute cutoff values. Nevertheless, the question remains what a model with all calculated goodness of fit indices in the acceptable range would look like. Associated with this question might be that Beck's cognitive model (1996) predicts cognitive variables are activated by an interaction of dysfunctional attitudes and stressors (e.g., life events and/or daily hassles). Thus, the omission of stressors from the model is an important limitation. Therefore, future research testing sequential interpretations of Beck's model should include measures of stressors.

The sole use of self-report instruments may be seen as another limitation of the current study. First, a mono-method bias of same informant and method for assessing all constructs in this study was likely. Second, the use of self-report instruments to measure cognitive variables representing a style of thinking (i.e., dysfunctional attitudes, cognitive distortions) may be criticized because it is questionable how much insight individuals really have into their own style of thinking (see Scher, Ingram, \& Segal, 2005, for a review). If such insight lies outside most individuals' awareness, information processing paradigms may be better suited than self-report questionnaires to measure style constructs. However, for cognitive distortions, for example, information processing paradigms have not yet been developed (Gotlib \& Neubauer, 2000). As self-report instruments already exist for all of the measured constructs, their use was deemed adequate for this study.

This study's restriction of the sample to a predominantly female university population provided homogeneity of age range, educational level, sex, and social environment, which may have limited the generalizability of the results other populations in general and to male populations in particular. Considering the differences in the prevalence rate of depression between men and women (Angst, Gamma, Gastpar, Lépine, Mendlewicz, \& Tylee, 2002) and the 
fact that some cognitive variables seem to be more prevalent in women than in men (for a review Nolen-Hoeksema, 2006), the generalizability of the findings to male populations was limited. However, we are aware of only two studies testing for possible differences in the associations between the cognitive variables as proposed by Beck's cognitive model (Beck, 1976, 1996; Beck \& Weishaar, 2005) and between the cognitive variables and depressive symptoms (Pössel, 2011; Pössel \& Thomas, 2011). Neither of the two studies found sex differences in these associations, making it more likely that our findings can be generalized to male populations. However, further research including more male participants is needed.

Further, the restriction to a sample without clinically significant depressive symptoms at Time 1 was purposeful to test the sequential interpretation of Beck's cognitive model of the development of depression (Beck, 1967, 1996; Beck \& Weishaar, 2005). However, one can see this restriction as a limitation, as the present findings cannot be generalized to individualswith clinically significant depressive symptoms. Thus, future studies with participants with clinically significant depressive symptoms are needed to test the sequential order of the cognitive variables in Beck's cognitive model of the maintenance of depression.

This study helped resolve the contradictory results found in previous studies (Burns \& Spangler, 2001; Joiner et al., 1999; Kwon \& Oei, 1992; Oei et al., 1996; Oei et al., 2005; Oei \& Kwon, 2007; Stewart et al., 2004) regarding the sequential order of Beck's cognitive model of the development of depression (Beck, 1976, 1996; Beck \& Weishaar, 2005). These studies tested the causal mediational and symptom interpretations of Beck's cognitive model but not the bidirectional model that integrates both models. Nevertheless, future research is needed that (a) includes more male participants, (b) includes individuals from a clinical sample, (c) includes 
measures of stressors, (d) includes at least four waves, and (e) employs different time lags between time points in order to test the stationarity assumption.

If replicated, the current findings have important consequences for the prevention of depression. Preventive interventions may focus on any cognitive construct and still impact all of the other cognitive constructs; however, a focus on cognitive distortions is the most promising because of their central position in the bidirectional network. This conclusion is supported by a study demonstrating that a cognitive-behavioral program focusing on cognitive distortions prevented the development of depressive symptoms in adolescents longer than other cognitivebehavioral programs focusing on other parts of the cognitive network (e.g., dysfunctional attitudes; Pössel, Adelson, \& Hautzinger, 2011). 


\section{References}

Alloy, L. B., Clements, C., \& Kolden, G. (1985). The cognitive diathesis-stress theories of depression: Therapeutic implications. In S. Reiss \& R. R. Bootzin (Eds.), Theoretical issues in behaviour therapy. Orlando, Fl.: Academic Press.

Angst, J., Gamma, A., Gastpar, M., Lépine, J.-P., Mendlewicz, J., \& Tylee, A. (2002). Gender differences in depression. Epidemiological findings from the European DEPRES I and II studies. European Archives of Psychiatry and Clinical Neuroscience, 252, 201 - 209.

Arbuckle, J. L. (1999). AMOS user's guide. Chicago, IL: SmallWaters.

Beck, A. T. (1967). Depression: Clinical, experimental, and theoretical aspects. New York, NY: Harper \& Row.

Beck, A. T. (1976). Cognitive therapy and the emotional disorders. New York, NY: International University Press.

Beck, A. T. (1996). Beyond belief: A theory of modes, personality, and psychopathology. In P. M. Salkovskis (Ed.), Frontiers of cognitive therapy. New York, NY: Guildford Press.

Beck, A. T. \& Weishaar, M. (2005). Cognitive therapy. In R. J. Corsini \& D. Wedding (Eds.), Current psychotherapies. Belmont, CA: Thomson Brooks.

Beckham, E. E., Leber, W. R., Watkins, J. T., Boyer, J. L., \& Cook, J. B. (1986). Development of an instrument to measure Beck's cognitive triad: The Cognitive Triad Inventory. Journal of Consulting and Clinical Psychology, 54, 566-567.

Bentler, P. M. (1990). Comparative fit indexes in structural models. Psychological Bulletin, 107, 238-246.

Brewin, C. R. (1985). Depression and causal relations: What is their relation? Psychological Bulletin, 98, 297-309. 
Burns, D. D. \& Spangler, D. L. (2001). Do changes in dysfunctional attitudes mediate changes in depression and anxiety in cognitive behavioral therapy? Behavior Therapy, 32, 337-369.

Butler, A. C., Chapman, J. E., Forman, E. M., \& Beck, A. T. (2006). The empirical status of cognitive-behavioural therapy: A review of meta-analyses. Clinical Psychology Review, $26,17-31$.

Chiu, S., Webber, M., Zeig-Owens, R., Gustave, J., Lee, R., Kelly, K., Rizzotto, L., \& Prezant, D. (2010). Validation of the CES-D scale in screening for major depressive disorders among retired firefighters exposed to the World Trade Center disaster. Journal of Affective Disorders, 121, 212-219.

Chou, C.-P. \& Bentler, P. M. (1995). Estimates and tests in structural equation modeling. In R. H. Hoyle (Ed.), Structural equation modeling: Concepts, issues and applications (pp. 3755). Thousand Oaks, CA: Sage.

Cole, D. A. \& Maxwell, S. E. (2003). Testing mediational models with longitudinal data: Questions and tips in the use of structural equation modelling. Journal of Abnormal Psychology, 112, 558-577.

Gotlib, I. H., \& Neubauer, D. L. (2000). Information-processing approaches to the study of cognitive biases in depression. In S. L. Johnson, A. M. Hayes, T. M. Field, N. Schneiderman, \& P. M. McCabe (Eds.), Stress, coping, and depression. Mahwah, NJ: Lawrence Erlbaum Associates.

Haringsma, A., Engels, G., Beekman, A., \& Spinhoven, P. (2004). The criterion validity of the CES-D in a sample of self-referred elders with depressive symptomatology. International Journal of Geriatric Psychiatry, 19, 558-563. 
Hautzinger, M., Bailer, M., Hofmeister, D., \& Keller, F. (2012). Allgemeine Depressions-Skala (ADS) (2nd ed.). Göttingen, Germany: Hogrefe.

Hautzinger, M., Joormann, J. \& Keller, F. (2005). Skala dysfunktionaler Einstellungen (DAS)Testhandbuch. Göttingen, Germany: Hogrefe.

Hollon, S. D., DeRubeis, R. J., \& Evans, M. D. (1996). Cognitive therapy in the treatment and prevention of depression In P. M. Salkovskis (Ed.), Frontiers of cognitive therapy. New York, NY: Guilford.

Hu, L. T. \& Bentler, P. M. (1998). Fit indices in covariance structure modeling: sensitivity to underparameterized model misspecification. Psychological Methods, 3, 424-453.

Hu, L., \& Bentler, P.M. (1999). Cutoff criteria for fit indexes in covariance structure analysis: Conventional criteria versus new alternatives. Structural Equation Modeling, 6, 1-55.

Ilardi, S. S., \& Craighead, W. E. (1999). Rapid early response, cognitive modification and nonspecific factors in cognitive behavior therapy for depression: a reply to Tang and DeRubeis. Clinical Psychology: Science and Practice, 6, 295-299.

Joiner, T. E., Jr., Metalsky, G. I., Lew, A., \& Klocek, J. (1999). Testing the causal mediation component of Beck's theory of depression: Evidence for specific mediation. Cognitive Therapy and Research, 23, 401-412.

Joreskog, K. G. \& Sorbom, D. (1993). LISREL 8: Structural equation modeling with the SIMPLIS command language. Chicago, IL: Scientific Software International.

Kendall, Ph. C., Howard, B. L, \& Hays, R. C. (1989). Self-referent speech and psychopathology: the balance of positive and negative thinking. Cognitive Therapy and Research, 13, 583598.

Kenny, D. A. (1979). Correlation and causality. New York, NY: Wiley. 
Kline, R. B. (2005). Principals and practice of structural equation modeling ( $2^{\text {nd }}$ ed.). New York, NY: Guilford.

Kwon, S.-M. \& Oei, T. P. S. (1992). Differential causal roles of dysfunctional attitudes and automatic thoughts in depression. Cognitive Therapy and Research, 16, 309-328.

Kwon, S.-M. \& Oei, T. P. S. (1994). The roles of two levels of cognitions in the development, maintenance, and treatment of depression. Clinical Psychology Review, 14, 331 - 358.

Lefebvre, M. F. (1981). Cognitive distortion and cognitive errors in depressed psychiatric and low back pain patients. Journal of Consulting and Clinical Psychology, 49, 517-525.

MacKinnon, D. P., Fritz, M. S., Williams, J., \& Lockwood, C. M. (2007). Distribution of the product confidence limits for the indirect effect: Program PRODCLIN. Behavior Research Methods, 39, 384-389.

Marsh, H. W., Hau, K. T., \& Wen Z. (2004). In search of golden rules: Comment on hypothesistesting approaches to setting cutoff values for fit indixes and dangers in overgeneralizing Hu and Bentler's (1999) findings. Structural Equation Modeling, 11, 320 - 341.

Meade, A. W., Johnson, E. C., \& Braddy, P. W. (2008). Power and sensitivity of alternative fit indices in tests of measurement invariance. Journal of Applied Psychology, 93, 568 592.

Martens, M. P. \& Haase, R. F. (2006). Advanced applications of Structural Equation Modeling in Counseling Psychology research. The Counseling Psychologist, 34, 878-911.

Nolen-Hoeksema, S. (2006). The etiology of gender differences in depression. In C. M. Mazure \& G. P. Keita (Eds.), Understanding depression in women: Applying empirical research to practice and policy. Washington, DC: American Psychological Association. 
Oei, T. P. S., Goh, Y.-W., \& Kwon, S.-M. (1996). A cross-sectional study of the integrated cognitive model of depression. Current Psychology of Cognition/Cahiers de psychologie cognitive, 15, 265-281.

Oei, T. P. S., Hibberd, E., \& O'Brien, A. J. (2005). Study of the integrated cognitive model of depression among Latin-Americans. Australian and New Zealand Journal of Psychiatry, 39, 932-939.

Oei, T. P. S. \& Kwon, S.-M. (2007). Evaluation of the integrated cognitive model of depression and its specificity in a migrant population. Depression and Anxiety, 24, 112-123.

Parry, G. \& Brewin, C. R. (1988). Cognitive style and depression: Symptom-related, eventrelated or independent provoking factor. British Journal of Clinical Psychology, 27, 2335.

Pössel, P. (2009a). Cognitive Error Questionnaire (CEQ): Psychometric properties and factor structure of the German translation. Journal of Psychopathology and Behavioural Assessment, 31, 264-269.

Pössel, P. (2009b). Cognitive Triad Inventory (CTI): Psychometric properties and factor structure of the German translation. Journal of Behavior Therapy and Experimental Psychiatry, 40, 240-247.

Pössel, P. (2011). Can Beck's theory of depression and the Response Style Theory be integrated? Journal of Counseling Psychology, 58, 618-629.

Pössel, P., Adelson, J., \& Hautzinger, M. (2011). A randomized trial to evaluate the course of effects of a program to prevent adolescent depressive symptoms over 12 months. Behaviour Research and Therapy, 49, 838-851. 
Pössel, P. \& Hautzinger M. (2006). Effekte pharmakologischer und psychotherapeutischer Interventionen auf Depressionen bei Kindern und Jugendlichen. [Effects of pharmacoand psychotherapeutic interventions of depression in children and adolescents.] Zeitschrift für Kinder- und Jugendpsychiatrie, 34, 243-255.

Pössel, P. \& Knopf, K. (2008). An experimental test of the maintenance and vulnerability hypothesis of depression in consideration of the cognitive hierarchy. Depression and Anxiety, 25, E47-E55.

Pössel, P., Seemann, S., \& Hautzinger, M. (2005). Evaluation eines deutschsprachigen Instrumentes zur Erfassung positiver und negativer automatischer Gedanken [Evaluation of a German instrument to measure positive and negative automatic thoughts]. Zeitschrift für Klinische Psychologie und Psychotherapie, 34, 27-34.

Pössel, P. \& Thomas, S. D. (2011). Cognitive Triad as mediator in the Hopelessness model? A short-term longitudinal study. Journal of Clinical Psychology, 67, 224-240.

Preacher, K. J., \& Hayes, A. F. (2008). Asymptotic and resampling strategies for assessing and comparing indirect effects in multiple mediator models. Behavior Research Methods, 40, 879-891.

Radloff, L. S. (1977). The CES-D: A self-report symptom scale to detect depression in the general population. Applied Psychological Measurement, 3, 385-401.

Scher, C. D., Ingram, R. E., \& Segal, Z. V. (2005). Cognitive reactivity and vulnerability: Empirical evaluation of construct activation and cognitive diatheses in unipolar depression. Clinical Psychology Review, 25, 487-510.

Steiger, J. H. \& Lind, J. M. (1980, May). Statistically based tests for the number of common factors. Paper presented at the Psychometrika Society meeting, Iowa City, IA. 
Stewart, S. M., Kennard, B. D., Lee, P. W. H., Hughes, C. W., Mayes, T. L., Emslie, G. J., \& Lewinsohn, P. M. (2004). A cross-cultural investigation of cognitions and depressive symptoms in adolescents. Journal of Abnormal Psychology, 113, 248-257.

Tucker, L. R. \& Lewis, C. (1973). The reliability coefficient for maximum likelihood factor analysis. Psychometrika, 38, 1-10.

Weissman, A.N., \& Beck, A.T. (1978). Development and validation of the Dysfunctional Attitude Scale (DAS). Presented at the 12th annual meeting of the Association for the Advancement of Behavior Therapy, Chicago, IL.

Wittchen, H.-U., Nelson, C. B., \& Lachner, G. (1998). Prevalence of mental disorders and psychosocial impairments in adolescents and young adults. Psychological Medicine, 28, 109-126. 
Table 1

Descriptive Data and Correlations between All Used Instruments.

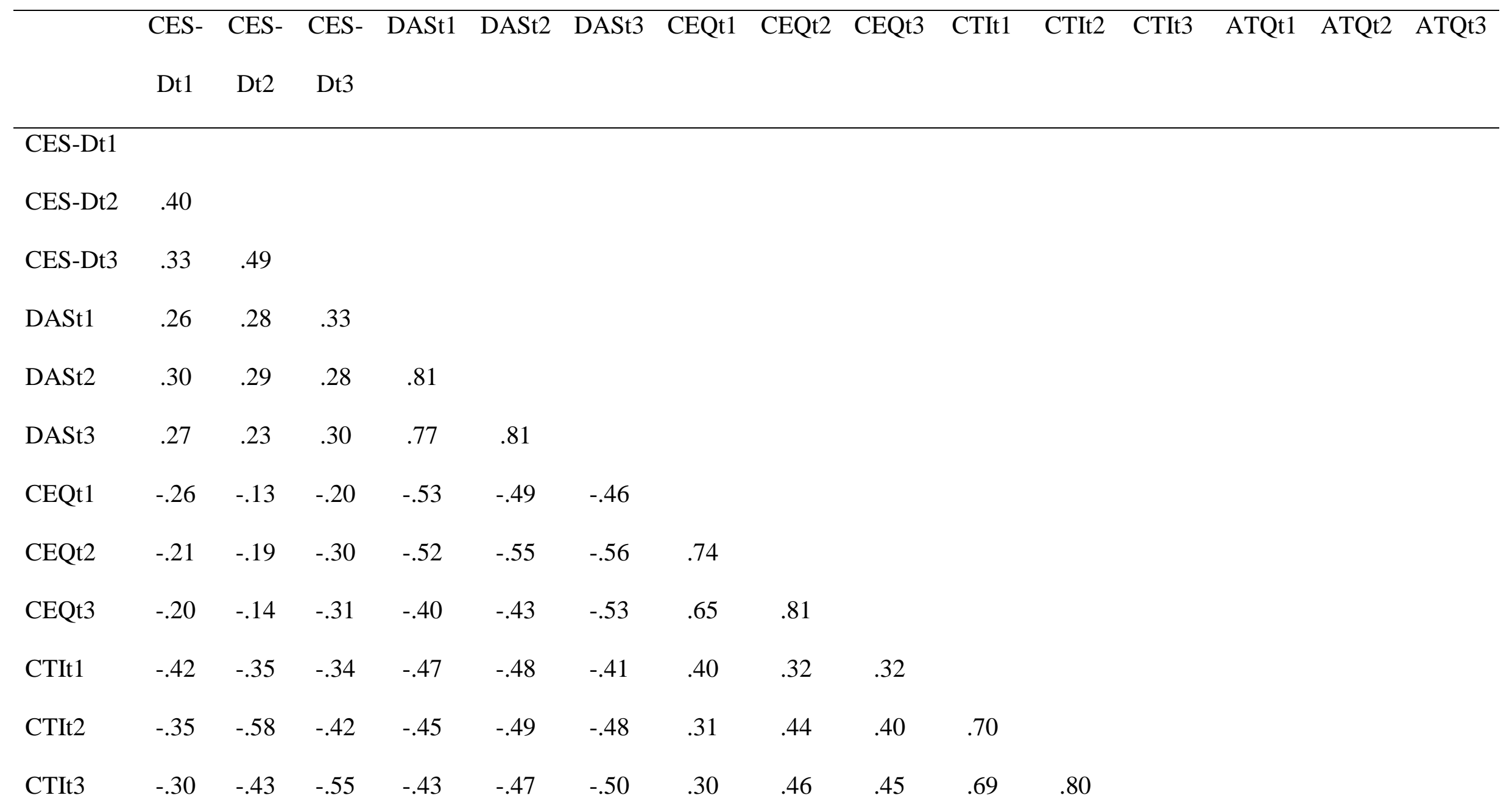


SEQUENTIAL INTERPRETATIONS OF BECK'S MODEL 33

\begin{tabular}{|c|c|c|c|c|c|c|c|c|c|c|c|c|c|c|c|}
\hline ATQt1 & .57 & .33 & .34 & .35 & .40 & .40 & -.36 & -.36 & -.35 & -.51 & -.43 & -.38 & & & \\
\hline ATQt2 & .32 & .72 & .41 & .44 & .42 & .39 & -.29 & -.36 & -.31 & -.42 & -.60 & -.49 & .57 & & \\
\hline ATQt3 & .30 & .34 & .72 & .35 & .26 & .38 & -.27 & -.36 & -.41 & -.34 & -.39 & -.52 & .60 & .53 & \\
\hline Mean & 11.25 & 13.28 & 15.07 & 97.00 & 94.89 & 96.18 & 75.15 & 76.29 & 76.78 & 166.18 & 166.31 & 165.89 & 18.86 & 21.53 & 22.02 \\
\hline$S D$ & 5.64 & 8.73 & 9.41 & 24.09 & 22.21 & 22.46 & 10.56 & 11.24 & 12.05 & 14.21 & 16.35 & 16.41 & 5.68 & 8.48 & 8.88 \\
\hline Range & $0-53$ & $0-52$ & $0-46$ & 4-186 & $45-$ & $34-$ & $36-96$ & $34-96$ & $16-96$ & $87-$ & 86- & $86-$ & $12-52$ & $12-51$ & $10-55$ \\
\hline & & & & & 164 & 173 & & & & 197 & 199 & 196 & & & \\
\hline
\end{tabular}

Note. $N \geq 235$ for all variables. CES-D = Center for Epidemiological Studies - Depression Scale; DAS = Dysfunctional Attitudes

Scale; CEQ = Cognitive Error Questionnaire; CTI = Cognitive Triad Inventory; ATQ = Automatic Thoughts Questionnaire, negative self-statements; $\mathrm{t} 1=$ time point $1 ; \mathrm{t} 2=$ time point $2 ; \mathrm{t} 3=$ time point 3 . All correlations are significant at $p<.001$. 
SEQUENTIAL INTERPRETATIONS OF BECK'S MODEL 34

Table 2

Indices of Goodness of Fit and Parsimony of the Tested Models.

\begin{tabular}{|c|c|c|c|c|c|c|}
\hline Models & $d f$ & $\chi^{2}$ & $\chi^{2} / d f$ & CFI & TLI & RMSEA \\
\hline Autoregressive model & 65 & 269.88 & 4.15 & .927 & .866 & .103 \\
\hline Causal meditational model with full mediation & 57 & 251.36 & 4.41 & .931 & .855 & .107 \\
\hline Symptom model with full mediation & 57 & 247.72 & 4.35 & .932 & .858 & .106 \\
\hline Bidirectional model with full mediation & 49 & 227.45 & 4.64 & .937 & .845 & .111 \\
\hline Causal meditational model with partial mediation & 45 & 198.04 & 4.40 & .946 & .855 & .107 \\
\hline Symptom model with partial mediation & 45 & 213.98 & 4.76 & .940 & .840 & .112 \\
\hline Bidirectional model with partial mediation & 25 & 143.47 & 5.74 & .958 & .798 & .126 \\
\hline
\end{tabular}

Note. $N=299$. Indices of goodness of fit or parsimony that are at least acceptable are in italics. CFI = Comparative Fit Index, TLI =

Tucker-Lewis Index; RMSEA = root mean square error of approximation. All $\chi^{2}$ are significant at $p<.05$. 
Table 3

Correlations of Constructs and Error Terms of the Constructs, Respectively, Within Each Time Point of the Bidirectional Model.

\begin{tabular}{|c|c|c|}
\hline DASt1 & CEQt1 & $-.550 * * *$ \\
\hline DASt1 & CTIt1 & $-.490 * * *$ \\
\hline DASt1 & ATQt1 & $.398 * * *$ \\
\hline DASt1 & CES-Dt1 & $.262 * * *$ \\
\hline CEQt1 & CTIt1 & $.402 * * *$ \\
\hline CEQt1 & ATQt1 & $-.374 * * *$ \\
\hline CEQt1 & CES-Dt1 & $-.265 * * *$ \\
\hline CTIt1 & ATQt1 & $-.514 * * *$ \\
\hline CTIt1 & CES-Dt1 & $-.426 * * *$ \\
\hline ATQt1 & CES-Dt1 & $.567 * * *$ \\
\hline DASt2error & CEQt2error & $-.230 * * *$ \\
\hline DASt2error & CTIt2error & $-.141 *$ \\
\hline DASt2error & ATQt2error & .083 \\
\hline DASt2error & CES-Dt2error & .061 \\
\hline CEQt2error & CTIt2error & $.376 * * *$ \\
\hline CEQt2error & ATQt2error & $-.139 *$ \\
\hline CEQt2error & CES-Dt2error & $-.125^{*}$ \\
\hline CTIt2error & ATQt2error & $-.458 * * *$ \\
\hline CTIt2error & CES-Dt2error & $-.490 * * *$ \\
\hline ATQt2error & CES-Dt2error & $.695 * * *$ \\
\hline
\end{tabular}




$\begin{array}{llc}\text { DASt3error } & \text { CEQt3error } & -.170^{* *} \\ \text { DASt3error } & \text { CTIt3error } & -.110+ \\ \text { DASt3error } & \text { ATQt3error } & .233 * * * \\ \text { DASt3error } & \text { CES-Dt3error } & .087 \\ \text { CEQt3error } & \text { CTIt3error } & .138^{*} \\ \text { CEQt3error } & \text { ATQt3error } & -.183 * * \\ \text { CEQt3error } & \text { CES-Dt3error } & -.129 * \\ \text { CTIt3error } & \text { ATQt3error } & -.388^{* * *} \\ \text { CTIt3error } & \text { CES-Dt3error } & -.422 * * * \\ \text { ATQt3error } & \text { CES-Dt3error } & .679 * * *\end{array}$

Note. CES-D = Center for Epidemiological Studies - Depression Scale; DAS = Dysfunctional Attitudes Scale; CEQ = Cognitive Error Questionnaire; CTI = Cognitive Triad Inventory; ATQ = Automatic Thoughts Questionnaire, negative self-statements; $\mathrm{t} 1=$ time point $1 ; \mathrm{t} 2$ = time point 2; $\mathrm{t} 3=$ time point 3 ; error $=$ error term. $+p<.10 ; * p<.05 ; * * p<.01 ; * * * p<.001$. 
Table 4

Total, Direct, and Indirect Effects and their 95\% Confidence Intervals testing for Multiple Mediations.

\begin{tabular}{|c|c|c|c|}
\hline & Effects & Lower CI & Upper CI \\
\hline \multicolumn{4}{|l|}{ Total effects } \\
\hline DASt1 - DASt2 & $.667 *$ & .571 & .756 \\
\hline DASt1 - CEQt2 & $-.062 *$ & -.129 & -.004 \\
\hline DASt1 - CTIt2 & $-.113^{*}$ & -.252 & -.020 \\
\hline DASt1 - ATQt2 & $.065 *$ & .027 & .110 \\
\hline DASt1 - CES-Dt2 & .054 & -.004 & .114 \\
\hline DASt1 - DASt3 & $.522 *$ & .416 & .627 \\
\hline DASt1 - CEQt3 & -.062 & -.130 & .003 \\
\hline DASt1 - CTIt3 & -.086 & -.218 & .005 \\
\hline DASt1 - ATQt3 & .037 & .003 & .079 \\
\hline DASt1 - CES-Dt3 & .041 & .004 & .094 \\
\hline DASt2 - DASt 3 & .742 & .627 & .853 \\
\hline DASt2 - CEQt3 & -.003 & -.063 & .054 \\
\hline DASt2 - CTIt3 & .018 & -.050 & .085 \\
\hline DASt2 - ATQt3 & -.010 & -.064 & .046 \\
\hline DASt2 - CES-Dt3 & .001 & -.059 & .064 \\
\hline CEQt1 - DASt2 & -.117 & -.292 & .069 \\
\hline CEQt1 - CEQt2 & $.675^{*}$ & .563 & .784 \\
\hline CEQt1 - CTIt2 & .005 & -.152 & .153 \\
\hline
\end{tabular}




\begin{tabular}{|c|c|c|c|}
\hline CEQt1 - ATQt2 & .026 & -.047 & .106 \\
\hline CEQt1 - CES-Dt2 & .076 & -.040 & .205 \\
\hline CEQt1 - DASt3 & $-.283^{*}$ & -.447 & -.126 \\
\hline CEQt1 - CEQt3 & $.569^{*}$ & .437 & .692 \\
\hline CEQt1 - CTIt3 & .146 & -.005 & .289 \\
\hline CEQt1 - ATQt3 & $-.088^{*}$ & -.177 & -.002 \\
\hline CEQt1 - CES-Dt3 & -.069 & -.171 & .027 \\
\hline CEQt2 - DASt3 & $-.268 *$ & -.471 & -.092 \\
\hline CEQt2 - CEQt3 & $.830^{*}$ & .721 & .941 \\
\hline CEQt2 - CTIt3 & $.198 *$ & .038 & .370 \\
\hline CEQt2 - ATQt3 & $-.147 *$ & -.273 & -.029 \\
\hline CEQt2 - CES-Dt3 & $-.147 *$ & -.295 & -.011 \\
\hline CTIt1 - DASt2 & $-.203^{*}$ & -.370 & -.034 \\
\hline CTIt1 - CEQt2 & -.003 & -.107 & .092 \\
\hline CTIt1 - CTIt2 & $.725^{*}$ & .529 & .865 \\
\hline CTIt1 - ATQt2 & -.063 & -.147 & .014 \\
\hline CTIt 1 - CES-Dt2 & $-.129 *$ & -.240 & -.024 \\
\hline CTIt1 - DASt3 & $-.163 *$ & -.316 & -.011 \\
\hline CTIt1 - CEQt3 & .016 & -.109 & .129 \\
\hline CTIt1 - CTIt3 & $.611^{*}$ & .434 & .762 \\
\hline CTIt1 - ATQt3 & -.028 & -.099 & .055 \\
\hline CTIt1 - CES-Dt3 & $-.110^{*}$ & -.188 & -.032 \\
\hline CTIt2 - DASt3 & -.045 & -.181 & .126 \\
\hline
\end{tabular}




\begin{tabular}{|c|c|c|c|}
\hline CTIt2 - CEQt3 & .038 & -.064 & .130 \\
\hline CTIt2 - CTIt3 & $.875^{*}$ & .756 & .990 \\
\hline CTIt2 - ATQt3 & -.003 & -.078 & .100 \\
\hline CTIt2 - CES-Dt3 & -.080 & -.177 & .026 \\
\hline ATQt1 - DASt2 & .202 & -.222 & .675 \\
\hline ATQt1 - CEQt2 & -.235 & -.486 & .013 \\
\hline ATQt1 - CTIt2 & -.085 & -.436 & .267 \\
\hline ATQt1 - ATQt2 & $.577^{*}$ & .318 & .803 \\
\hline ATQt1 - CES-Dt2 & .085 & -.223 & .393 \\
\hline ATQt1 - DASt3 & .395 & .001 & .824 \\
\hline ATQt1 - CEQt3 & $-.290 *$ & -.522 & -.055 \\
\hline ATQt1 - CTIt3 & -.035 & -.386 & .316 \\
\hline ATQt1 - ATQt3 & $.375^{*}$ & .185 & .563 \\
\hline ATQt1 - CES-Dt3 & .076 & -.112 & .274 \\
\hline ATQt2 - DASt3 & .356 & -.004 & .706 \\
\hline ATQt2 - CEQt3 & $-.181^{*}$ & -.376 & -.002 \\
\hline ATQt2 - CTIt3 & .131 & -.172 & .449 \\
\hline ATQt2 - ATQt3 & $.604 *$ & .403 & .801 \\
\hline ATQt2 - CES-Dt3 & .001 & -.263 & .244 \\
\hline CES-Dt1 - DASt2 & -.001 & -.396 & .430 \\
\hline CES-Dt1 - CEQt2 & .103 & -.108 & .325 \\
\hline CES-Dt1 - CTIt2 & -.123 & -.411 & .188 \\
\hline CES-Dt1 - ATQt2 & -.057 & -.233 & .154 \\
\hline
\end{tabular}




\begin{tabular}{|c|c|c|c|}
\hline CES-Dt1 - CES-Dt2 & $.397 *$ & .160 & .656 \\
\hline CES-Dt1 - DASt3 & -.170 & -.518 & 201 \\
\hline CES-Dt1 - CEQt3 & .155 & -.026 & .346 \\
\hline CES-Dt1 - CTIt3 & -.064 & -.315 & .201 \\
\hline CES-Dt1 - ATQt3 & -.079 & -.198 & .040 \\
\hline CES-Dt1 - CES-Dt3 & $.155^{*}$ & .007 & .314 \\
\hline CES-Dt2 - DASt3 & -.319 & -.624 & .011 \\
\hline CES-Dt2 - CEQt3 & $.162 *$ & .023 & .299 \\
\hline CES-Dt2 - CTIt3 & .079 & -.142 & .329 \\
\hline CES-Dt2 - ATQt3 & -.075 & -.232 & .064 \\
\hline CES-Dt2 - CES-Dt3 & $.403^{*}$ & .202 & .609 \\
\hline \multicolumn{4}{|l|}{ Direct effects } \\
\hline DASt1 - DASt2 & $.667^{*}$ & .571 & .756 \\
\hline DASt1 - CEQt2 & $-.062 *$ & -.129 & -.004 \\
\hline DASt1 - CTIt2 & $-.113 *$ & -.252 & -.020 \\
\hline DASt1 - ATQt2 & $.065^{*}$ & .027 & .110 \\
\hline DASt1 - CES-Dt2 & .054 & -.004 & .114 \\
\hline DASt2 - DASt3 & $.742 *$ & .627 & .853 \\
\hline DASt2 - CEQt3 & -.003 & -.063 & .054 \\
\hline DASt2 - CTIt3 & .018 & -.050 & .085 \\
\hline DASt2 - ATQt3 & -.010 & -.064 & .046 \\
\hline DASt2 - CES-Dt3 & .001 & -.059 & .064 \\
\hline CEQt1 - DASt2 & -.117 & -.292 & .069 \\
\hline
\end{tabular}




\begin{tabular}{|c|c|c|c|}
\hline CEQt1 - CEQt2 & $.675^{*}$ & .563 & .784 \\
\hline CEQt1 - CTIt2 & .005 & -.152 & .153 \\
\hline CEQt1 - ATQt2 & .026 & -.047 & .106 \\
\hline CEQt1 - CES-Dt2 & .076 & -.040 & .205 \\
\hline CEQt2 - DASt3 & $-.268^{*}$ & -.471 & -.092 \\
\hline CEQt2 - CEQt3 & $.830^{*}$ & .721 & .941 \\
\hline CEQt2 - CTIt3 & $.198^{*}$ & .038 & .370 \\
\hline CEQt2 - ATQt3 & $-.147 *$ & -.273 & -.029 \\
\hline CEQt2 - CES-Dt3 & $-.147 *$ & -.295 & -.011 \\
\hline CTIt1 - DASt2 & $-.203^{*}$ & -.370 & -.034 \\
\hline CTIt1 - CEQt2 & -.003 & -.107 & .092 \\
\hline CTIt1 - CTIt2 & $.725^{*}$ & .529 & .865 \\
\hline CTIt1 - ATQt2 & -.063 & -.147 & .014 \\
\hline CTIt1 - CES-Dt2 & $-.129 *$ & -.240 & -.024 \\
\hline CTIt2 - DASt3 & -.045 & -.181 & .126 \\
\hline CTIt2 - CEQt3 & .038 & -.064 & .130 \\
\hline CTIt2 - CTIt3 & $.875^{*}$ & .756 & .990 \\
\hline CTIt2 - ATQt3 & -.003 & -.078 & .100 \\
\hline CTIt2 - CES-Dt3 & -.080 & -.177 & .026 \\
\hline ATQt1 - DASt2 & .202 & -.222 & .675 \\
\hline ATQt1 - CEQt2 & -.235 & -.486 & .013 \\
\hline ATQt1 - CTIt2 & -.085 & -.436 & .267 \\
\hline ATQt1 - ATQt2 & $.577^{*}$ & .318 & .803 \\
\hline
\end{tabular}




\begin{tabular}{|c|c|c|c|}
\hline ATQt1 - CES-Dt2 & .085 & -.223 & .393 \\
\hline ATQt2 - DASt3 & .356 & -.004 & .706 \\
\hline ATQt2 - CEQt3 & $-.181 *$ & -.376 & -.002 \\
\hline ATQt2 - CTIt3 & .131 & -.172 & .449 \\
\hline ATQt2 - ATQt3 & $.604 *$ & .403 & .801 \\
\hline ATQt2 - CES-Dt3 & .001 & -.263 & .244 \\
\hline CES-Dt1 - DASt2 & -.001 & -.396 & .430 \\
\hline CES-Dt1 - CEQt2 & .103 & -.108 & .325 \\
\hline CES-Dt1 - CTIt2 & -.123 & -.411 & .188 \\
\hline CES-Dt1 - ATQt2 & -.057 & -.233 & .154 \\
\hline CES-Dt1 - CES-Dt2 & $.397 *$ & .160 & .656 \\
\hline CES-Dt2 - DASt3 & -.319 & -.624 & .011 \\
\hline CES-Dt2 - CEQt3 & $.162^{*}$ & .023 & .299 \\
\hline CES-Dt2 - CTIt3 & .079 & -.142 & .329 \\
\hline CES-Dt2 - ATQt3 & -.075 & -.232 & .064 \\
\hline CES-Dt2 - CES-Dt3 & $.403^{*}$ & .202 & .609 \\
\hline \multicolumn{4}{|l|}{ Indirect effects } \\
\hline DASt1 - DASt3 & $.522^{*}$ & .416 & .627 \\
\hline DASt1 - CEQt3 & -.061 & -.130 & .003 \\
\hline DASt1 - CTIt3 & -.086 & -.218 & .005 \\
\hline DASt1 - ATQt3 & $.037^{*}$ & .003 & .079 \\
\hline DASt1 - CES-Dt3 & $.041 *$ & .004 & .094 \\
\hline CEQt1 - DASt3 & $-.283 *$ & -.447 & -.126 \\
\hline
\end{tabular}




\begin{tabular}{lccc} 
CEQt1 - CEQt3 & $.569^{*}$ & .437 & .692 \\
CEQt1 - CTIt3 & .146 & -.005 & .289 \\
CEQt1 - ATQt3 & $-.088^{*}$ & -.177 & -.002 \\
CEQt1 - CES-Dt3 & -.069 & -.171 & .027 \\
CTIt1 - DASt3 & $-.163^{*}$ & -.316 & -.011 \\
CTIt1 - CEQt3 & .016 & -.109 & .129 \\
CTIt1 - CTIt3 & $.611^{*}$ & .434 & .762 \\
CTIt1 - ATQt3 & -.028 & -.099 & .055 \\
CTIt1 - CES-Dt3 & $-.110^{*}$ & -.188 & -.032 \\
ATQt1 - DASt3 & $.395^{*}$ & .001 & .824 \\
ATQt1 - CEQt3 & $-.290^{*}$ & -.522 & -.055 \\
ATQt1 - CTIt3 & -.035 & -.386 & .316 \\
ATQt1 - ATQt3 & $.375^{*}$ & .185 & .563 \\
ATQt1 - CES-Dt3 & .076 & -.112 & .274 \\
CES-Dt1 - DASt3 & -.170 & -.518 & .201 \\
CES-Dt1 - CEQt3 & .155 & -.026 & .346 \\
CES-Dt1 - CTIt3 & -.064 & -.315 & .201 \\
CES-Dt1 - ATQt3 & -.079 & -.198 & .040 \\
CES-Dt1 - CES-Dt3 & $.155^{*}$ & .007 & .314 \\
\hline
\end{tabular}

Note. A statistically significant mediation effect exists when the $95 \%$ confidence interval do not contain zero. $* p<.05$ 
SEQUENTIAL INTERPRETATIONS OF BECK'S MODEL 44

Table 5

Confidence Intervals for all Possible Mediation Effects in the Bidirectional Model.

Lower Confidence Limit Upper Confidence Limit

\begin{tabular}{llc}
\hline Bidirectional model & & $.020^{*}$ \\
DASt1 - CEQt2 - ATQt3 & .001 & $.021^{*}$ \\
DASt1 - CEQt2 - CES-Dt3 & .001 & $.149 *$ \\
ATQt1 - CEQt2 - DASt3 & .002 & .001 \\
CEQt1 - CTIt2 - ATQt3 & -.001 & .016 \\
CTIt1 - ATQt2 - CES-Dt3 & -.016 & .024 \\
CTIt1 - CEQt2 - DASt3 & -.022 & .010 \\
ATQt1 - CTIt2 - CEQt3 & -.021 & \\
\hline
\end{tabular}

Note. A statistically significant mediation effect exists when the $95 \%$ confidence interval do not contain zero. $* p<.05$ 
Figure 1. Causal interpretations of Beck's cognitive model (1976) with full mediation.

Figure 2. Path diagrams of each of the tested structural equation models. Solid lines represend paths in the models with full mediation, dashed lines represend paths in the models with partial mediations.

Figure 3. Structural equational model of the bidirectional model. All constructs and error terms of the constructs, respectively, measured at one time point are correlated. These correlations are not pictured for reasons of clarity but can be found in Table $3 . \mathrm{t} 1=$ time point $1 ; \mathrm{t} 2=$ time point $2 ; \mathrm{t} 3=$ time point $3 ;+p<.10 ; * p<.05 ; * * p<.01 ; * * * p<.001$. 